\title{
A Novel Distance Estimation Algorithm for Bluetooth Devices Using RSSI
}

\author{
Junhua Huang, Song Chai ${ }^{*}$, Nan Yang and Liang Liu \\ The fourth part of the ring road No.16, Wuhou District, Chengdu, Sichuan, China \\ ${ }^{*}$ Corresponding author
}

\begin{abstract}
A lot of proximity related Bluetooth applications, such as Bluetooth indoor positioning/navigation and proximity detection, has emerged on market by using Received Signal Strength Index(RSSI). One of the key technology in these application is to estimate distance between Bluetooth devices. In this paper, a distance estimation algorithm is proposed for Bluetooth devices. The RSSI values are first processed using median filter, and then converted to distance values. Finally, Kalman filtering is applied to further reduce noise. Our experiments show that our algorithm has average accuracy of 0.1 0.4m.
\end{abstract}

Keywords-bluetooth, distance estimation; RSSI, median filter; Kalman fitler

\section{INTRODUCTION}

In recent years, Bluetooth Low Energy(LE) has being widely used in short-range wireless communication[1]. Bluetooth LE allows designers to inquire Received Signal Strength Index (RSSI), which indicates radio signal power level received by antenna[2], and it enables a blooming market of proximity related application, such as indoor positioning[3], indoor navigation[4], indoor tracking[5], proximity detection[6] and so on.

The key problem in such proximity related Bluetooth applications is to calculated distance between Bluetooth devices, known as distance estimation[7], and most distance estimation algorithm for Bluetooth devices are implemented based on RSSI.

In our paper, a novel distance estimation algorithm is proposed for Bluetooth device. First, the received RSSI values are filtered using median filter to eliminate any outliers. Then the processed RSSI are converted to distance values. Finally, further noise reduction is performed by applying Kalman filter.

The rest of this paper is organized as follow: Section 2 discuss some related work in proximity based Bluetooth applications; our algorithm is proposed in Section 3; Section 4 shows the experiment results; Section 5 concludes the paper.

\section{RELATED WORK}

By inquiring RSSI in Bluetooth LE, various proximity related Bluetooth applications are proposed in recent years. An indoor positioning system is proposed in [3], which uses Euclidean distance correction algorithm to calibrate distance estimation. In [4], a positioning and navigation system is proposed for indoor scenario using Bluetooth devices. The location of Bluetooth device is determined by calculating distance between current device and surrounding Bluetooth devices, and navigation is implemented using grid following method. A Bluetooth pedestrian indoor tracking system is proposed in [5]. The system uses Bayesian filter to predict further movement, and results in an $80 \mathrm{~cm}$ accuracy.

\section{PROPOSED ALGORITHM}

\section{A. Median Filter}

RSSI received by Bluetooth device is corrupted due to severely multipath fading in indoor scenario[8], shown as blue line in Figure 1. Most RSSI values lie between $-65 \mathrm{~dB}$ and $-68 \mathrm{~dB}$ with some outlier falling below $-72 \mathrm{~dB}$. Thus these outliers must be removed before further process.

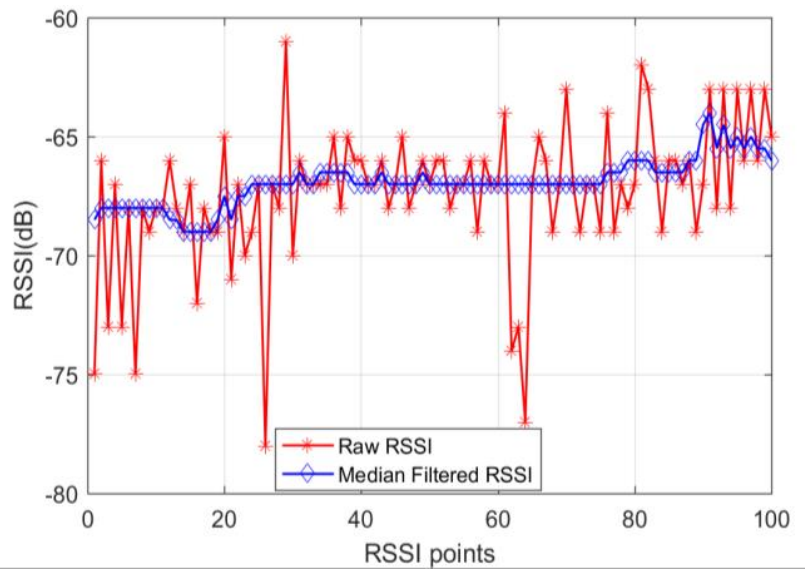

FIGURE I. RAW RSSI MEASURED AT 1M DISTANCE AND MEDIAN FILTERED RSSI

RSSI outliers are removed by using median filter [9] in our algorithm. Ten most recent RSSI values are collected as RSSI_raw, and sorted as RSSI_sorted. The $[R S S I$ _sorted $(5)+R S S I$ _sorted $(6)] \div 2$, namely median of RSSI_sorted, is rendered for future process, noted as RSSI_median. The result of median filtering is shown as blue line in Figure 1.

\section{B. Distance Calculation}

RSSI_median is then converted to distance value. During the conversion, the RSSI measured in $1 \mathrm{~m}$ distance, known as the reference RSSI[10] (RSSI_refer), is needed. Then the distance is calculated using Equation 1, which is obtained by first measuring RSSI in varies distance, and then fitting the data into the experience formula. 


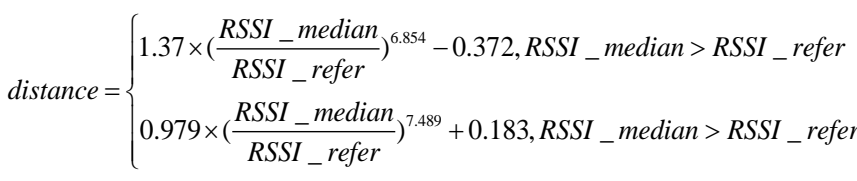

\section{Kalman Filtering}

To further reduce noise in calculated distance in previous section, Kalman filter is applied to increase the accuracy of estimated distance[11]. The flow of Kalman filter is shown in Figure 2.

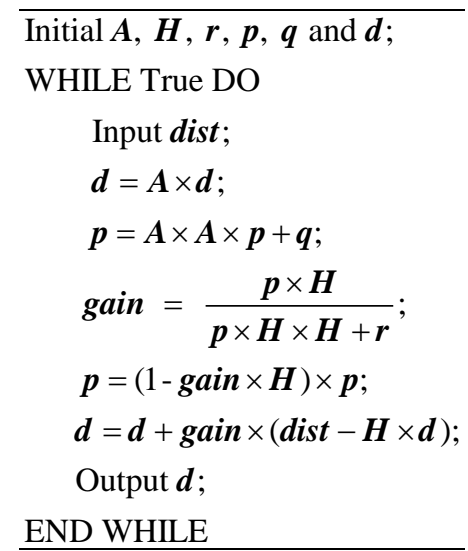

FIGURE II. FLOW OF KALMAN FILTER

\section{EXPERIMENT AND RESULT}

In our experiment, the distance between two Bluetooth devices, which both are Texas Instrument CC2541 Development Board, is evaluated using our distance estimation algorithm. A new RSSI is observed every $0.1 \mathrm{~s}$, and the distance between two devices is evaluated every $0.1 \mathrm{~s}$ too.

Figure 3 (a) (d) shows the stationary distance estimation of $0.6 \mathrm{~m}, 1 \mathrm{~m}, 2 \mathrm{~m}$ and $3 \mathrm{~m}$ respectively. As observed in these figures, our algorithm has a solid performance within $2 \mathrm{~m}$ distance, with average error of $0.1 \mathrm{~m}$. When increasing distance to $3 \mathrm{~m}$, as shown in Figure 3(d), the average error boost to $0.41 \mathrm{~m}$.

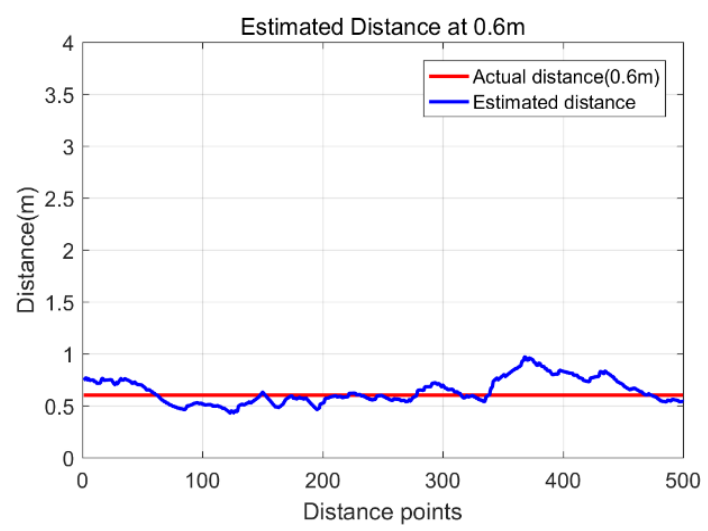

(a)

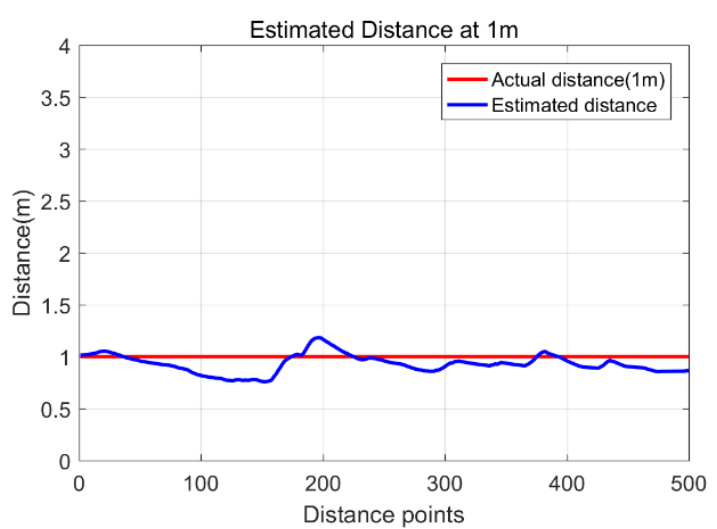

(b)

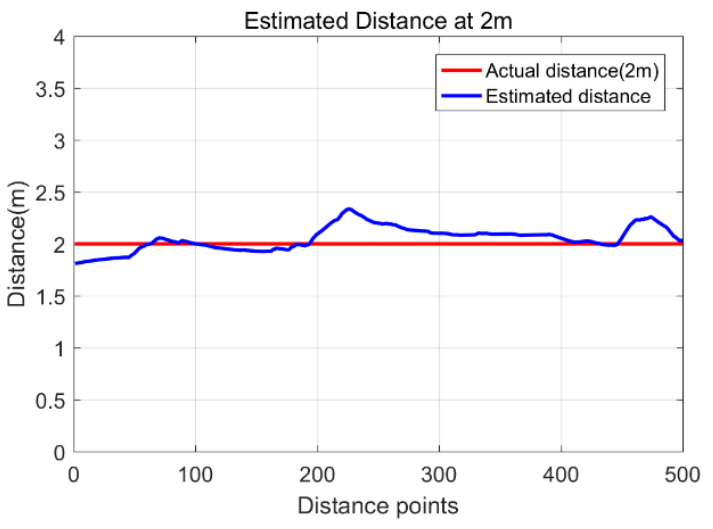

(c)

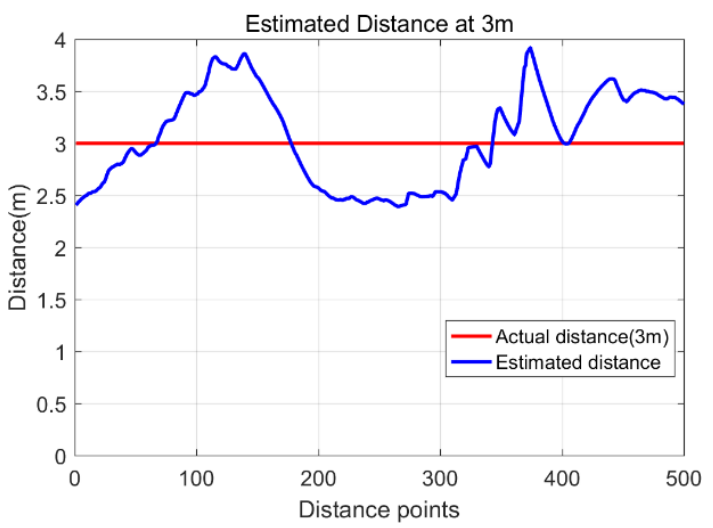

(d)

FIGURE III. STATIONARY RESULTS

Figure 4 shows the results of dynamic scenario, in which two Bluetooth devices moves away from $2 \mathrm{~m}$ to $5 \mathrm{~m}$, and then move together from $5 \mathrm{~m}$ to $2 \mathrm{~m}$ with constant velocity. In figure 4 , red line represents the actual distance and blue line is the estimated distance. As observed in this figure, estimated distance always follows actual distance with a lag of 5 points $(0.5 \mathrm{~s})$, which is introduced in the median filtering stage. 


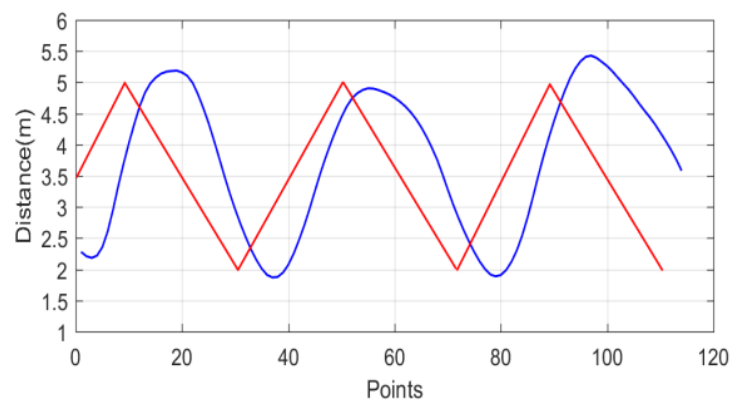

FIGURE IV. RESULTS OF DYNAMIC SCENARIO

\section{CONCLUSION}

A distance estimation algorithm is proposed for Bluetooth devices in this paper. The algorithm first uses median filter to remove RSSI outliers, and then converts RSSI to distance values. Finally, Kalman filtering is applied to further reduce noise. Experiment results show that our algorithm has average accuracy of $0.1 \sim 0.4 \mathrm{~m}$.

\section{REFERENCE}

[1] Treurniet, J. J, et al. "Energy Consumption and Latency in BLE Devices under Mutual Interference: An Experimental Study." The, International Conference on Future Internet of Things and Cloud IEEE, 2015:333-340.

[2] Fang, Hao, et al. "A novel adaptive algorithm for location based on Distance-Loss model in complex environment." IEEE, International Conference on Computer Supported Cooperative Work in Design IEEE, 2016:26-30.

[3] Wang, Yankai, et al. "Indoor positioning system using Euclidean distance correction algorithm with bluetooth low energy beacon."International Conference on Internet of Things and Applications IEEE, 2016:243-247.

[4] Sudhakar, Kumbhar Shrikant, B. A. Dnyanoba, and P. Ramchandran "Protocol Development for Indoor Navigation using Bluetooth Device."Indian Journal of Science \& Technology 8.21(2015).

[5] Bao, Zhenshan, L. Wang, and W. Zhang. "Bayesian Filtering for Bluetooth RSS-based Indoor Tracking." International Conference on Intelligent Control and Computer Application Atlantis Press, 2016.

[6] Park, J., Y. K. Cho, and S. K. Timalsina. "Direction Aware Bluetooth Low Energy Based Proximity Detection System for Construction Work Zone Safety." Proceedings of the, International Symposium on Automation and Robotics in Construction 2016.

[7] Roy, Prottay Kanti, and R. Kumar. "Accurate Estimation of Bluetooth RSSI and Distance." International Journal of Engineering \& Technical Research V5.3(2016).

[8] Hamdoun, Safa, A. Rachedi, and A. Benslimane. "RSSI-based Localization Algorithms using Spatial Diversity in Wireless Sensor Networks." International Journal of Ad Hoc \& Ubiquitous Computing 19.3/4(2014):263-266.

[9] Deak, Gabriel, K. Curran, and J. Condell. "Filters for RSSI-based measurements in a Device-free Passive Localisation Scenario." Image Processing \& Communications 15(2011):23-34.

[10] Zang, Yan Hong, et al. "The Hybrid HMM for RSS-Based Localization in Wireless Sensor Networks." Applied Mechanics \& Materials 347-350(2013):796-802.

[11] Jung, Joon Young, et al. "D2D distance measurement using Kalman filter algorithm for distance-based service in an office environment."International Conference on Advanced Communication TechnologyIEEE, 2015:221-224. 